\title{
Prompt Photons at HERA and TEVATRON
}

\author{
Vladislav Šimák* \\ Czech Technical University, Czech Republic \\ E-mail: simakefzu.cz
}

\section{Sijbrand de Jorg; Vladimir Chekelian§̧ Matthew Wing}

Teh following short report summarizes results on prompt photon production at the Tevatron experiment, D0, and the HERA experiments, H1 and ZEUS.

The D0 Collaboration presents a measurement of the cross section for isolated photons in the $P_{T}$ range from 22 to $300 \mathrm{GeV}$, for the central rapidity region $|\eta|<0.9$. This analysis uses $330 \mathrm{pb}^{-1}$ of integrated luminosity which was gathered between 2002 and 2004 at a center of mass energy of $1.96 \mathrm{TeV}$. The results are compared with next to leading order QCD predictions[1].

The H1 experiment presents a measurement of photoproduction of isolated prompt photons, inclusively and associated with jets in the $\gamma p$ center of mass energy range $142<W<266 \mathrm{GeV}$. The cross sections are measured for transverse momentum of the photons and associated jets: $E_{T}^{j e t}>4.5 \mathrm{GeV}, E_{T}^{\gamma}>5 \mathrm{GeV}$. They are measured differentially as a function of $E_{T}^{\gamma}$, $E_{T}^{j e t}$, the pseudorapidity $\eta^{\gamma}$ and $\eta^{j e t}$ and estimators of the momentum fractions $x_{\gamma}$ and $x_{p}$ of the incident photon and proton carried by the constituents participating in the hard process. In order to further investigate the underlying dynamics, the angular correlation between the prompt photon and the jet in the transverse plane is studied. Comparisons with the predictions of the event generators PYTHIA and HERWIG are also presented[2].

The ZEUS experiment has also measured prompt photon production inclusively and with an accompanying jet in deep inelastic scattering. The results are compared to next to leading order QCD predictions and Monte Carlo models[3].

International Europhysics Conference on High Energy Physics

July 21st - 27th 2005

Lisboa, Portugal

\footnotetext{
*Speaker.

${ }^{\dagger}$ D0 Collaboration

Radboud University Nijmegen/NIKHEF, D0 Colaboration

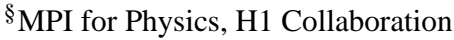

IIDESY/UCL, ZEUS Collaboration
} 


\section{Isolated Photon Cross Section at 1.96 TeV, Experiment Do}

In the D0 experiment[4], photon candidates were selected using the following criteria. Each electromagnetic (EM) cluster-photon candidate is formed by a simple cone algorithm with the cone size or $\mathcal{R}=\sqrt{\Delta \eta^{2}+\Delta \phi^{2}}=0.2$. The data were initially preselected with the requirement of at least one EM cluster with $p_{T}>15 \mathrm{GeV}$. Candidate EM clusters were accepted within the pseudorapidity region $|\eta|<0.9$. Each candidate was required to deposit more than $95 \%$ of the detected energy in the EM section of the calorimeter and to be isolated in the annular region between $\mathcal{R}=0.2$ and $\mathcal{R}=0.4$ around the energy-weighted centroid. An artificial neural network (ANN) for pattern recognition was used. The events that have network output value greater than 0.5 were selected (see Fig.1). Since the signal events cannot be identified on an event-by-event basis, their photon

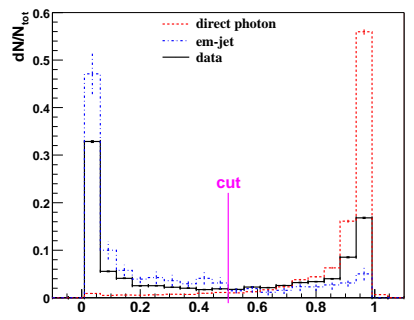

Figure 1: Distribution of ANN output for data.

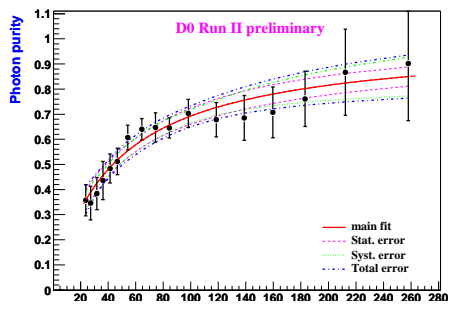

Figure 2: Dependence of the photon purity on $p_{T}^{\gamma}$.

fraction (purity) $\mathcal{P}$ is determined for a given $p_{T}^{\gamma}$ bin statistically. The photon purity is defined as ratio $\mathcal{P}=N^{\gamma} /\left(N^{\gamma}+N^{\text {jet }}\right)$, where $N^{\gamma}\left(N^{\text {jet }}\right)$ is the number of single photons (QCD jets) that passed the selection criteria (see Fig.2.). The total number of photon candidates remaining after the analysis of the initial data set collected with $L_{i n t}=325.9 \pm 21.2 \mathrm{pb}^{-1}$ and application of all the selection criteria is about 2.7 million. The cross section and ratio Data/Theory are shown in Fig. 3

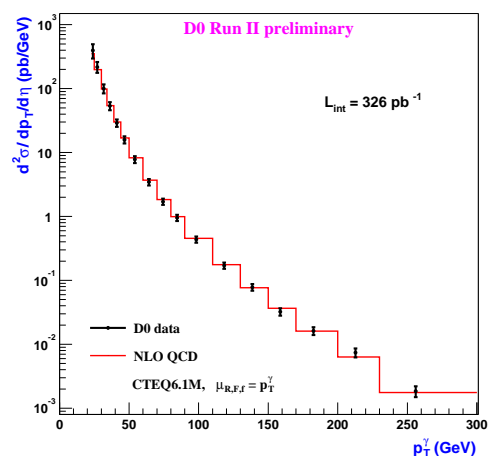

Figure 3: The inclusive cross section for production of isolated photon vs. $p_{T}^{\gamma}$.

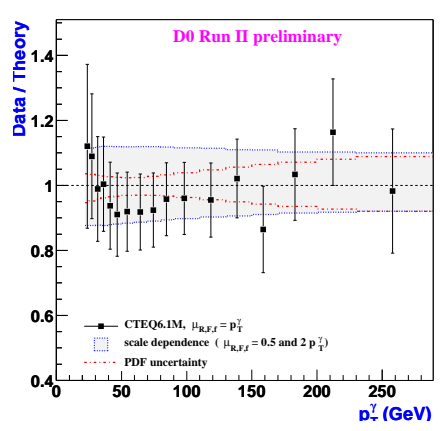

Figure 4: The ratio of the measured cross section to the NLO QCD predictions vs. $p_{T}^{\gamma}$.

and Fig.4. as a function of $p_{T}^{\gamma}$. One can see that in the range $23<p_{T}^{\gamma}<300 \mathrm{GeV}$, the photon cross section falls by about 5 orders of magnitude. The uncertainty in the measurement is dominated at low $p_{T}^{\gamma}$ by the systematics which vary between 11 and $25 \%$.

The NLO QCD predictions, both with CTEQ6.1M and MRST2004, describe the data within the experimental uncertainties in the whole $p_{T}^{\gamma}$ range considered. 


\section{Measurement of Prompt Photon Cross Sections in Photoproduction at HERA, Experiment $\mathrm{H1}$}

Photons are identified in the H1 liquid argon (LAr) calorimeter as compact electromagnetic clusters with no track associated to them. The data were collected in the years 1996-2000 with the $\mathrm{H} 1$ detector at HERA in data taking periods where electrons or positrons with energy $E_{e}=$ $27.6 \mathrm{GeV}$ collided with protons of energies $E_{p}=820 \mathrm{GeV}$ or $E_{p}=920 \mathrm{GeV}$ [5]. The data corresponds to an integrated luminosity of $105 \mathrm{pb}^{-1}$ of which $28.8 \mathrm{pb}^{-1}$ and $61.3 \mathrm{pb}^{-1}$ were recorded in $e^{+} p$ interactions at center of mass energies $\sqrt{s}=301 \mathrm{GeV}$ and $\sqrt{s}=319 \mathrm{GeV}$, respectively, and $14.9 \mathrm{pb}^{-1}$ were recorded in $e^{-} p$ interactions at $\sqrt{s}=319 \mathrm{GeV}$.

The results are presented in Fig.5. for $5<E_{T}^{\gamma}<10 \mathrm{GeV},-1<\eta^{\gamma}<0.9, E_{T}^{\text {cone }}<0.1 E_{T}^{\gamma}$. The inner error bars on the data points in the figures indicate the statistical errors as obtained from the shower discrimination fits. The full error bars contain the systematic errors added in quadrature. Differential cross sections $d \sigma / d E_{T}^{\gamma}$ and $d \sigma / d \eta^{\gamma}$ for inclusive prompt photon production are compared with the predictions of the PYTHIA and HERWIG event generators.

Predictions by perturbative QCD calculations in next to leading order are about $30 \%$ below the inclusive prompt photon data after corrections for hadronisation and multiple interactions, but are in reasonable agreement with the results for prompt photons associated with jets.
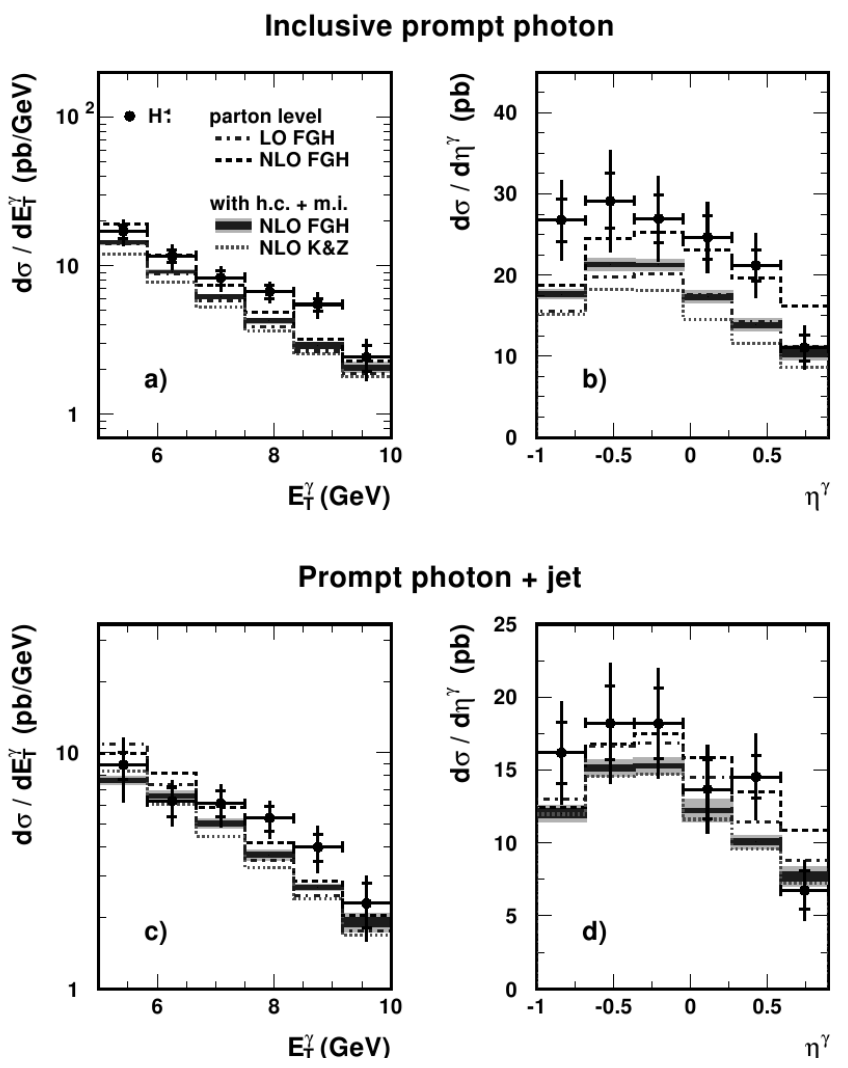

Figure 5: Inclusive prompt photon cross sections $(\mathrm{a}, \mathrm{b})$ and with additional jet requirement $(\mathrm{c}, \mathrm{d})$. The data are compared with NLO pQCD. 


\section{Study of Prompt Photons in Deep Inelastic Scattering at HERA, Experiment ZEUS}

For the ZEUS analysis [6] a data sample corresponding to an integrated luminosity of $122 p b^{-1}$ was used, taken between 1996 and 2000.

The cross section for inclusive prompt photon production, $e p \rightarrow e \gamma X$, has been measured in following kinematic region: $Q^{2}>35 \mathrm{GeV}^{2}, E_{e}>10 \mathrm{GeV}, 139.8^{\circ}<\theta_{e}<171.8^{\circ},-0.7<\eta^{\gamma}<0.9$ and $5<E_{T}^{\gamma}<10 \mathrm{GeV}$, with photon isolation such that at least $90 \%$ of energy found in an $\eta-\phi$ cone of radius 1.0 around the photon is associated with the photon. The measured cross section is

$$
\sigma(e p \rightarrow e \gamma X)=5.64 \pm 0.58(\text { stat. })_{-0.72}^{+0.47}(\text { syst. }) p b .
$$

The predicted cross section prom PYTHIA and HERWIG are lower than the data by factors of approximately 2 and 8, respectively. The higher measured cross section compared to MC seems to be compatible with the observation in the experiment H1. Both PYTHIA and HERWIG describe the $E_{T}^{\gamma}$ spectrum normalized to the data and HERWIG describes the rapidity well.

The measured total cross section for photon plus a single jet within this kinematic region $\left(E_{T}^{j e t}>6 \mathrm{GeV}\right.$ and $\left.-1.5<\eta^{j e t}<1.8\right)$ is

$$
\sigma(e p \rightarrow e \gamma+j e t+Y)=0.86 \pm 0.14(\text { stat } .)_{-0.34}^{+0.44}(\text { syst } .) p b .
$$

The results have been compared to an $O\left(\alpha^{3} \alpha_{s}\right)$ parton-level calculation for $e p \rightarrow e \gamma+j e t+Y$ in the acceptance region of this measurement. The level of agreement is satisfactory in photon rapidity and jet transverse energy but only fair for photon transverse energy and jet pseudorapidity. The total predicted cross section is consistent with the measured value.

\section{References}

[1] Sijbrand de Jong, HEP2005, abstract 245

[2] Vladimir Chkelian, HEP2005, abstract 561

[3] Mattew Wing, HEP2005, abstract 373

[4] The Isolated Photon Cross Section in Central Rapidity Region at $1.96 \mathrm{TeV}$, D0 Collaboration, D0note 4859-CONF, 2005.

[5] Measurement of Prompt Photon Cross Sections in Photoproduction at HERA. H1 Collaboration, ISSN 0418-9833, July 2004, arXiv:hep-ex/0407018 v1

[6] Observation of isolated high- $E_{T}$ photons in deep inelastic scattering, ZEUS Collaboration, DESY-04-16, May 2004. 